ANNALS OF

CLINICAL

NEUROPHYSIOLOGY

\title{
Miller Fisher syndrome in a patient with pulmonary tuberculosis
}

\author{
Jae Young Park, Hoe Jong Jung, Heewon Bae, Jeong-Ho Han, Min Ju Kang \\ Department of Neurology, Veterans Health Service Medical Center, Seoul, Korea
}

Received: July 30, 2020

Revised: August 28, 2020

Accepted: September 1, 2020

Miller Fisher syndrome (MFS) is characterized by the acute ophthalmoparesis, ataxia and areflexia. We describe the case of 70-year-old man with cardinal symptom of MFS and active pulmonary tuberculosis (Tb). A thorough evaluation led to the diagnosis of MFS and treatment with intravenous immunoglobulin (IVlg) was started. The complete resolution of ophthalmoparesis and ataxia was observed from the fourth day of IVIg treatment. This is the first report to describe a case of MFS that developed in patient pulmonary tuberculosis.

Key words: Miller Fisher syndrome; Tuberculosis; Guillain-Barré syndrome

\section{Correspondence to}

\section{Min Ju Kang}

Department of Neurology, Veterans Health Service Medical Center, 53 Jinhwangdo-ro 61-gil, Gangdong-gu, Seoul 05368, Korea Tel: $+82-2-2225-4354$

Fax: +82-2-2225-1327

E-mail:minju0321@naver.com

ORCID

Jae Young Park

https://orcid.org/0000-0001-8641-3786

Hoe Jong Jung

https://orcid.org/0000-0002-8724-4184

Heewon Bae

https://orcid.org/0000-0002-2266-9288

Jeong-Ho Han

https://orcid.org/0000-0003-3197-4898

Min Ju Kang

https://orcid.org/0000-0002-7736-6073

Miller Fisher syndrome (MFS) is a variant of Guillain-Barré syndrome (GBS) that is characterized by the acute onset of ophthalmoparesis, ataxia, and areflexia. ${ }^{1}$ The pathomechanism underlying MFS is acute immune-mediated polyneuropathy, and two-thirds of cases are preceded by infection.' Numerous reports on tuberculosis and GBS have been published, but the association between these two diseases remains unclear., ${ }^{2,3}$ Although tuberculosis is widespread in Asia, there have been few reports of patients with GBS and tuberculosis, ${ }^{4}$ and no cases of MFS combined with tuberculosis have been reported. Here we report a rare case of MFS associated with pulmonary tuberculosis.

\section{CASE}

A 70-year-old male visited the emergency room complaining of horizontal diplopia and gait disturbance, which had first appeared on the previous day. He also had a 1-week history of sputum production, cough, and fever. He had a past medical history of chronic obstructive pulmonary disease. A physical examination revealed a fever of $38^{\circ} \mathrm{C}$ and a bronchial breathing sound with coarse crepitations, which was further confirmed in chest computed tomography (Fig. 1). A neurologic examination revealed left ptosis, limitation of depression, and slight limitation of adduction in the left eye (Fig. 2A), mild dysarthria, ataxia of both the upper and lower limbs, and areflexia of both lower limbs. Although his mus- 
cle strength was intact, proprioceptive deficits were noted in both extremities. The patient was unable to ambulate.

Brain magnetic resonance imaging (MRI) with gadolinium contrast scans revealed no abnormalities. Blood test results, including the complete blood count and electrolyte levels, were normal except for elevation of C-reactive protein at $3.85 \mathrm{mg} / \mathrm{L}$. Cerebrospinal fluid (CSF) analysis revealed albuminocytologic dissociation: opening pressure, $90 \mathrm{mmH}_{2} \mathrm{O}$; protein, $66 \mathrm{mg} / \mathrm{dL}$; glucose, $75 \mathrm{mg} / \mathrm{dL}$; white blood cells,
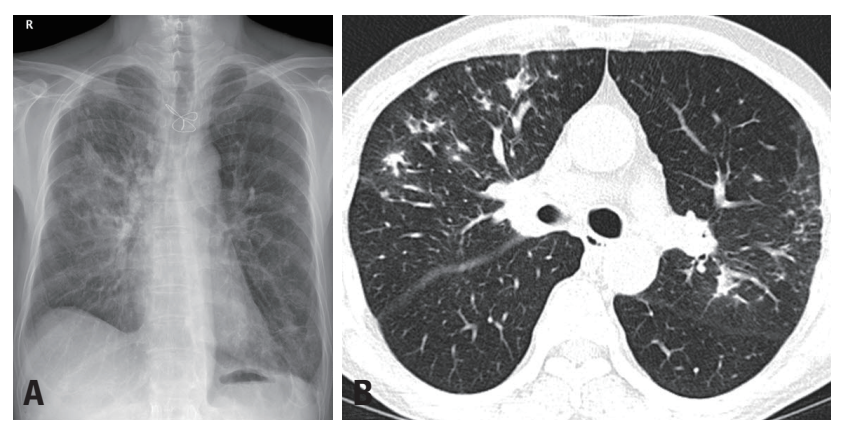

Fig. 1. Initial chest X-ray (A) and low-dose chest computed tomography scan (B) showing patch consolidations and diffuse nodules in both upper lobes, suggesting active pulmonary tuberculosis.
17/ $\mu \mathrm{L}$, red blood cells, 0/mL; adenosine deaminase, $1 \mathrm{IU} / \mathrm{L}$; and blood glucose, $99 \mathrm{mg} / \mathrm{dL}$. An MRI scan of the lumbar spine revealed mild degenerative changes with no other abnormalities. The results of sputum acid-fast bacillus staining and the mycobacterium tuberculosis polymerase chain reaction were positive, and so pulmonary tuberculosis was diagnosed.

The patient was started on antituberculous therapy comprising isoniazid, rifampin, ethambutol, and pyrazinamide along with $50 \mathrm{mg}$ of prednisolone. However, the ataxia continued to deteriorate over the following 3 days. Nerve conduction studies (NCS) and needle electromyography (EMG) were performed at 7 days after symptom onset. The NCS revealed slow motor conduction velocities and absent F-wave responses in the ulnar, peroneal, and tibial nerves. Both motor and sensory latencies were prolonged and the amplitudes were either reduced or borderline in bilateral ulnar and peroneal nerves. EMG revealed no abnormalities. Compound muscle action potentials were normal in both facial nerves. Repetitive nerve stimulation and acetylcholine receptor antibody testing produced normal results. An enzyme-linked immunosorbent assay to detect various
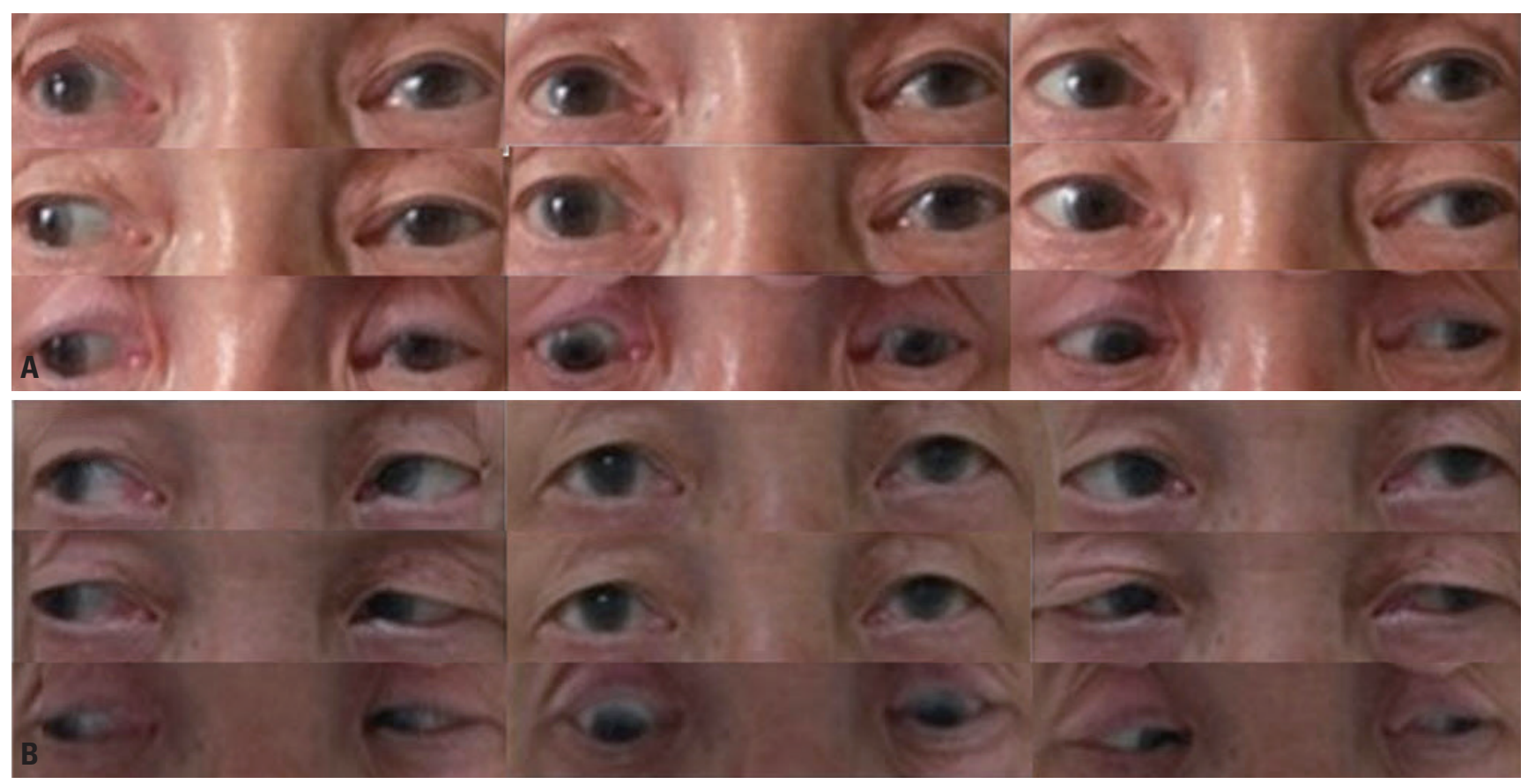

Fig. 2. Extraocular movement in nine cardinal gazes: (A) at 7 days after symptom onset there were left ptosis, limitation of depression, and slight limitation of adduction in the left eye; and (B) at 14 days after symptom onset there were improvements in the left ptosis and extraocular movement limitation. The patient consented to the use of these photographs. 
antiganglioside antibodies for immunoglobulin $\mathrm{G}$ and $\mathrm{M}$ antibodies against GM1, GD1a, and GQ1b produced negative results. Vitamin $B 1$ and $B 12$ levels were normal. Based on the electrophysiologic findings, laboratory results, and clinical presentation, the patient was diagnosed with MFS, and treatment with intravenous immunoglobulin (IVlg) $(0.4 \mathrm{~g} / \mathrm{kg}$, daily) was started.

We observed marked improvements in ophthalmoparesis and ataxia from the fourth day of IVlg treatment, which was discontinued on the fifth day of treatment due to the side effect of thrombocytopenia. Full ocular movement in both eyes (Fig. 2B) as well as the ambulation ability were restored at 7 days after initiating the IVlg treatment. The patient was discharged 2 weeks later after a further 4-day course of IVlg treatment. At a 4-week follow-up, neither ophthalmoparesis nor ataxia was observed.

\section{DISCUSSION}

The clinical presentation and test results of the current case led to a diagnosis of MFS associated with pulmonary tuberculosis. Because limb ataxia and ophthalmoparesis were prominent in this case, the possibility of meningoencephalitis or radiculomyelitis - which are common neurologic manifestations of tuberculosis ${ }^{4}$ - was initially considered. However, the involvement of the brainstem and spinal cord was ruled out by nonspecific MRI findings. The patient presented a triad of MFS symptoms (ataxia, ophthalmoparesis, and areflexia) and showed marked improvements in neurologic deficits after IVIg treatment. Although the findings for the GQ1b antibody were negative, CSF and electrophysiologic investigations supported the diagnosis of MFS.

GBS is associated with an autoimmune response in peripheral-nerve and spinal roots. The GBS classification is based on clinical and electrophysiologic features, and is supported by the identification of a specific antibody directed against neuronal membrane gangliosides; that is, GQ1 $b^{5}{ }^{5}$ Similar to GBS, MFS has been reported to occur as a result of infections, most of which affect the respiratory tract. ${ }^{6} \mathrm{~A}$ large case-control study showed that Campylobacter jejuni and Haemophilus influenzae are the main antecedent infectious pathogens of MFS. 'However, there have been no previous reports of pulmonary tuberculosis-associated MFS.
The pathogenesis of GBS associated with tuberculosis is unknown, but it is believed to occur due to molecular mimicry that leads to an immunologic attack on the peripheral and cranial nerves. ${ }^{3}$ Antigens may be produced in response to tuberculosis proteins or cytokines that are generated as a result of tuberculosis treatment. A delayed hypersensitivity reaction or invasion of the nerve roots caused by tuberculosis is another possible explanation for the associated neuropathy. ${ }^{8}$ Most cases of GBS manifest as acute inflammatory demyelinating polyneuropathy and acute motor axonal neuropathy, and these cases also exhibit features suggestive of tuberculosis even prior to the onset of weakness. ${ }^{2,9}$ The high prevalence of pulmonary tuberculosis suggests that GBS is also prevalent, but this remains unrecognized or untreated, like in other neurologic manifestations of tuberculosis.

Considering the timing of MFS and systemic symptoms of pulmonary tuberculosis, it is highly likely that pulmonary tuberculosis was the cause of MFS in the present case. The patient showed the complete recovery of neurologic deficits after receiving treatment for pulmonary tuberculosis. Therefore, the possibility of combined MFS and pulmonary tuberculosis should be considered in pulmonary tuberculosis patients who experience a complication of ataxia or ophthalmoparesis.

\section{Acknowledgements}

This study was supported by a VHS Medical Center research grant (Republic of Korea) (Grant No. VHSMC20037).

\section{Conflict of Interest}

The authors have no financial conflict of interest.

\section{REFERENCES}

1. Yuki N, Hartung HP. Guillain-Barré syndrome. N Engl J Med 2012;366:2294-2304.

2. Mohta S, Soneja M, Vyas S, Khot W. Tuberculosis and Guillain-Barré syndrome: a chance association? Intractable Rare Dis Res 2017;6:55-57.

3. Malakar S, Sharma TD, Raina S, Sharma KN, Kapoor D. Guillain Barre syndrome with pulmonary tuberculosis: a case series from a tertiary care hospital. J Family Med Prim Care 2019;8:1794-1797. 
4. Garg RK. Tuberculosis of the central nervous system. Postgrad Med J 1999;75:133-140.

5. Arányi Z, Kovács T, Sipos I, Bereczki D. Miller Fisher syndrome: brief overview and update with a focus on electrophysiological findings. Eur J Neurol 2012;19:15-20, e1-e3.

6. Yuki N. Infectious origins of, and molecular mimicry in, Guillain-Barré and Fisher syndromes. Lancet Infect Dis 2001;1:29-37.

7. Koga M, Gilbert M, Li J, Koike S, Takahashi M, Furukawa K, et al.
Antecedent infections in Fisher syndrome: a common pathogenesis of molecular mimicry. Neurology 2005;64:1605-1611.

8. Vyravanathan S, Senanayake N. Guillain-Barré syndrome associated with tuberculosis. Postgrad Med J 1983;59:516-517.

9. Singh A, Balasubramanian V, Gupta N. The association of Guillain-Barré syndrome with pulmonary tuberculosis: chance or causal? Neurol India 2020;68:209-218. 